\title{
Electrochemical Characteristics of Gatifloxacin and Its Interaction with DNA
}

\author{
Ming GuO*广 and Qing-Sen Y $\mathrm{U}^{* *}$ \\ * Department of Chemistry, Zhejiang Forestry University, LinAn 311300, China \\ **Department of Chemistry, Zhejiang University, Hangzhou 310027, China
}

\begin{abstract}
The electrochemical behavior of gatifloxacin (GTFX) and its interaction with natural calf thymus DNA (ctDNA) is investigated by differential pulse voltammetry (DPV) on a carbon paraffined electrode. According to the suggested electrochemical equation, a binding constant of $1.7058 \times 10^{5}\left(\mathrm{~mol} \mathrm{~L}^{-1}\right)^{-1}$ and binding sizes $s=3.09$ (base pairs) of GTFX with ctDNA are obtained by nonlinear fit analysis of electrochemical data. The results demonstrate that GTFX has the properties of an intercalative binder.
\end{abstract}

(Received September 5, 2005; Accepted December 15, 2005)

\section{Introduction}

Gatifloxacin (1-cyclopropyl-6-fluoro-1,4-dihydro-8-methoxy-7(3-methyl-1-piperazinyl)-4-oxo-3-quinolinecarboxylic acid, GTFX) belongs to the family of fluoroquinolone antibacterial agents. GTFX remains more active than similar fluoroquinolones or macrolides. It is one of the most potent quinolone derivatives in clinical use, with a wide spectrum of activity against Gram-positive cocci and some anaerobic bacteria. ${ }^{1,2}$ The drug is well tolerated and the intake of food has no adverse effect on the pharmacokinetic features. The risk of photo toxicity is also considered low. ${ }^{3}$

Many investigations on the interaction of drug molecules with DNA have been carried, ${ }^{4-10}$ such as UV, fluorescence, circular dichroism, NMR, Raman and calorimetry. The results of these various binding studies are very useful in designing new and promising drugs for clinical use and in developing sensitive chemical probes of nucleic acid structure. Drug of quinolone has also been investigated by various techniques. The earlier studies include an electrochemical method using $\mathrm{Hg}$ electrode, but the solid paraffined graphite electrode is not described. It is necessary to investigate the characteristics of GTFX and its interaction with DNA at the solid paraffined graphite electrode. Such studies will be important in providing basic electroactive information of GTFX at this electrode, and the results of binding data between GTFX and ctDNA will provide a measurable explanation for the binding degree of GTFX-DNA. Understanding the interaction between GTFX and DNA may also help to elucidate the mechanism of action of this important class of antibacterial agents, and may ultimately lead to the design of better, more potent antibacterial agents with fewer side effects. In this paper, our focus is to determine the interaction of GTFX-ctDNA using a solid electrode; to our knowledge, few attempts have been made to clarify such interaction.

\footnotetext{
$\dagger$ To whom correspondence should be addressed.

E-mail: tdky@hotmail.com
}

\section{Principle of Binding Equations for Drug-binding Studies}

The electrochemical equations can be deduced from McGhee's theory ${ }^{11}$ as well as other published materials, ${ }^{6,12-15}$ the following is a simple introduction.

The interaction of targeted drugs binding to DNA is regarded as an interaction of ligand-receptor. We will follow this suggestion for constructing a possible model: ${ }^{11}$ (i) The DNA molecule is taken as a linear array of $L$ units (lattice); the basic lattice residue can correspond to a nucleic acid base-pair. For convenience, we take a specific case in which the lattice is polar and oriented in a particular direction with respect to the observer (e.g. $3^{\prime} \rightarrow 5^{\prime}$ from left to right, for a single-stranded nucleic). (ii) The ligand, namely the target drug, is also taken as polar, and is assumed to bind with a fixed orientation with respect to the lattice, and to cover $s$ consecutive lattice residues. Initially we will also consider the lattice to be infinitely long; operationally this means $L \gg s$, and thus end-effects can be neglected. (iii) The precedence of ligand binding to lattice is not taken into account, and the partial binding by the ligand, resulting in "dangling" or "looping", is not allowed. (iv) A free residue of receptor is labeled as $f$, and a bound residue is labeled b. Any bound ligand can be divided into $n$ sections, each one corresponding to the underlying lattice residue; we number these sections, $1,2, \ldots, n$. Thus, for our present purposes a ligand binding site consists of any $s$ consecutive free lattice residues.

The association of an electroactive ligand ET $\left(C_{\mathrm{t}}\right.$, the total concentration of ligand; $C_{\mathrm{b}}$, the binding concentration of ligand; $C_{\mathrm{f}}$, the free concentration of ligand) with a binding site (S) is composed of $s$ base pairs (the total receptor concentration is $C_{\mathrm{DNA}}$, the receptor concentration in each unit is $C_{\mathrm{bp}}$ ) on a DNA duplex, to form a complex (ET-S), and the average number of ligands along a DNA (receptor) molecule can be described as $Z$ (i.e. binding density). Ligand-ligand interaction is only allowed between the nearest neighbors, bound without interveining free lattice residues. This restriction results in three distinguishable types of ligand binding sites. (i) An "isolated" site to which a 
ligand binds with an intrinsic association constant, $K\left(\mathrm{M}^{-1}\right)$. The two nearest neighbors (left and right sites) to this kind of site are naked. The concentration of binding ligand in this "isolated" site is $C_{\text {bisol }}$, and the concentration of this site, which is free and could be binding with ligand is $C_{\text {siol }}$; (ii) A "single contiguous" site to which a ligand binds with an association constant $K \omega$ $\left(\mathrm{M}^{-1}\right)$. One nearest neighbor (left or right sites) to this kind of site is naked, and the other neighbor is a ligand binding site. The concentration of binding ligand in this "single contiguous" site is $C_{\mathrm{b}_{\mathrm{s}}}$, and the concentration of this site, which is free and could be binding with ligand, is $C_{\mathrm{s} w}$; (iii) A "double contiguous" site to which a ligand binds with an association constant $K \omega^{2}$ $\left(\mathrm{M}^{-1}\right)$. The two nearest neighbors (left and right sites) to this kind of site are ligand binding sites. The concentration of binding ligand in this "isolated" site is $C_{\mathrm{b}_{\mathrm{k}} \text {, and the }}$ concentration of this site, which is free and could be binding with ligand, is $C_{\mathrm{S}_{\mathrm{sc}}}$. The cooperative parameter $\omega$ is the (unitless) equilibrium constant for the process of moving a bound ligand from an isolated site to a single contiguous site, or from a single contiguous site to a double contiguous site. For $\omega>1$, the ligands attract each other and the binding is positively cooperative; for $\omega<1$, the ligands repel each other and the binding is anti or negatively cooperative; for $\omega=1$, the binding is noncooperative.

A procedure to obtain a binding equation that meets such preconditions can be outlined now. For any particular stretch of $s$ consecutive free lattice residues, the binding equilibrium may be written as:

$$
\text { Free ligand binding site }+ \text { Free ligand } \stackrel{K}{\rightleftharpoons} \text { Bound ligand }
$$

For which the mass-action equation (square brackets denoting concentration) is:

$$
\frac{[\text { Bound ligand }]}{[\text { Free ligand }]}=K[\text { Free ligand binding sites }]
$$

The concentration of "free ligand binding sites" is symbolized as $C_{\mathrm{s}}$, and the microscopic equilibrium constant for binding is

$$
C_{\mathrm{b}} / C_{\mathrm{f}}=K C_{\mathrm{s}}
$$

We write the mass-action equation appropriate to each type of site as:

$$
\begin{aligned}
& C_{\mathrm{b}_{\mathrm{iso}}} / C_{f}=K(\text { average number of free isolated binding } \\
& \text { sites per lattice })=K C_{\text {siol }} \\
& C_{\mathrm{bu}} / C_{\mathrm{f}}=K \text { (average number of free single contiguous } \\
& \text { binding sites per lattice })=K \omega C_{\mathrm{s}_{\mathrm{c}}} \\
& C_{\mathrm{b}_{\mathrm{d}}} / C_{\mathrm{f}}=K \text { (average number of free double contiguous } \\
& \text { binding sites per lattice) }=K \omega^{2} C_{S_{d u}}
\end{aligned}
$$

The following results will be reached by adding the left and right sides in Eq. (1).

$$
\frac{C_{\mathrm{b}_{\mathrm{ivo}}}+C_{\mathrm{b}_{\mathrm{sc}}}+C_{\mathrm{b}_{\mathrm{bc}}}}{C_{\mathrm{f}}}=K\left(C_{\mathrm{sisal}}+\omega C_{\mathrm{ssc}}+\omega^{2} C_{\mathrm{S}_{\mathrm{dc}}}\right)
$$

With an established system, the following relationship could be made

$$
C_{\mathrm{b}_{\mathrm{ssol}}}+C_{\mathrm{b}_{\mathrm{sc}}}+C_{\mathrm{b}_{\mathrm{dc}}}=C_{\mathrm{b}}
$$

Consider the $Z$ value, for the average number of bound ligand per receptor under a particular experimental condition, the following equation is yielded by definition:

$$
C_{\mathrm{b}}=Z C_{\mathrm{DNA}}
$$

Then, the following results could be generalized:

$$
\frac{Z C_{\mathrm{DNA}}}{C_{\mathrm{f}}}=K\left(C_{\mathrm{sisol}_{\mathrm{sol}}}+\omega C_{\mathrm{ssc}_{\mathrm{c}}}+\omega^{2} C_{\mathrm{S}_{\mathrm{dc}}}\right)
$$

We can express these average numbers of isolated binding sites, single contiguous binding sites and double contiguous binding sites in per lattice as $Q_{\text {isol }}, Q_{\mathrm{sc}}, Q_{\mathrm{dc}}$, respectively; this can lead to the following results:

$$
\begin{aligned}
& C_{\mathrm{S}_{\mathrm{isol}}}=Q_{\mathrm{isol}} C_{\mathrm{DNA}} \\
& C_{\mathrm{scc}}=Q_{\mathrm{sc}} C_{\mathrm{DNA}} \\
& C_{\mathrm{sdc}}=Q_{\mathrm{dc}} C_{\mathrm{DNA}}
\end{aligned}
$$

When Eq. (5) is introduced into Eq. (4), then the $C_{\mathrm{DNA}}$ can be canceled out:

$$
\frac{Z}{C_{\mathrm{f}}}=K\left(Q_{\text {isol }}+\omega Q_{\mathrm{sc}}+\omega^{2} Q_{\mathrm{dc}}\right)
$$

While the average number of bound ligands to per receptor molecule is $Z$, there are average numbers of $Z+1$ gaps to the receptor, and any particular gap between adjacent bound ligands is exactly $g$ free lattice residues long. We could write conditional probabilities, $P_{\mathrm{g}}$, in this notation as:

$$
P_{\mathrm{g}}=\left(b_{\mathrm{s}} f\right)(f f)^{(\mathrm{g}-1)}\left(f b_{1}\right)
$$

Here, $f b_{1}$ is the probability, given a free lattice residue (i.e. an $f$ residue), that the left end of a bound ligand (i.e. a $b_{1}$ residue) lies on the immediate right. $b_{s} f$ is the probability, if one had selected the right end of a bound ligand (i.e. a $b_{\mathrm{s}}$ residue), that the lattice residue to the immediate right is free (i.e. an $f$ residue). $f f$ is the probability, given a free residue, that there is a second free residue to the immediate right.

We next obtain an expression for the average number of each of the three types of free binding sites per gap in terms of $P_{\mathrm{g}}$ and the following considerations: if $g<s$, there are no free binding sites; if $g=s$, there is one double contiguous binding site; if $g \geqslant$ $s+1$ there are two single contiguous binding sites (i.e. one at each end of the gap) and finally if $g \geqslant s+2$, there are $(g-s-1)$ isolated binding sites per gap. Thus, we obtain:

$$
\begin{aligned}
& S_{\text {isol }}=\sum_{\mathrm{g}=\mathrm{s}+2}^{\infty}(g-s-1) P_{\mathrm{g}}=\left(b_{\mathrm{s}} f\right)(f f)^{\mathrm{s}+1} /\left(f b_{1}\right) \\
& S_{\mathrm{sc}}=\sum_{\mathrm{g}=\mathrm{s}+1}^{\infty} 2 P_{\mathrm{g}}=2\left(b_{\mathrm{s}} f\right)(f f)^{\mathrm{s}} \\
& S_{\mathrm{dc}}=1 \cdot P_{\mathrm{s}}=\left(b_{\mathrm{s}} f(f f)^{\mathrm{s}-1}\left(f b_{1}\right)\right.
\end{aligned}
$$

While $L \rightarrow \infty, Z+1 \approx Z$, then these formulae could be shown,

$$
\begin{aligned}
& Q_{\text {isol }}=(Z+1) S_{\text {isol }} \approx Z S_{\text {isol }} \\
& Q_{\mathrm{sc}}=(Z+1) S_{\mathrm{sc}} \approx Z S_{\mathrm{sc}} \\
& Q_{\mathrm{dc}}=(Z+1) S_{\mathrm{dc}} \approx Z S_{\mathrm{dc}}
\end{aligned}
$$

From Eq. (6), the result could be reached:

$$
1 / C_{\mathrm{f}}=K\left(b_{\mathrm{s}} f\right)(f f)^{\mathrm{s}-1}\left((f f)^{2} /\left(f b_{1}\right)+2 \omega(f f)+\omega^{2}\left(f b_{1}\right)\right)
$$

In response to the assumption condition, these relationships should exist between various conditional probabilities. 


$$
\begin{aligned}
& (f f)+\left(f b_{1}\right)=1 \\
& \left(b_{s} f\right)+\left(b_{\mathrm{s}} b_{1}\right)=1
\end{aligned}
$$

Here, $b_{\mathrm{s}} b_{1}$ stands for the probability, having selected the right end of a bound ligand (i.e. a $b_{\mathrm{s}}$ residue), that the left end of a bound ligand (i.e. a $b_{1}$ residue) lies to the immediate right.

Consider a point on the equilibrium-binding isotherm at which there are, on average, $Z$ ligands bound to the lattice. The total number of lattice sites is then $(s Z)$, and the fraction of covered sites (the number of binding lattices/the total number of lattice) is $s Z / L$; the concentration format can be expressed as $s C_{\mathrm{b}} / C_{\mathrm{bp}}$. Hence the fraction of all lattice residues which are free is $(1-$ $s Z)$. Equivalently, $(1-s Z)$ is also the probability that a lattice residue selected at random is free. Similarly, the concentration format is $\left(1-s C_{\mathrm{b}} / C_{\mathrm{bp}}\right)$.

In addition, if one considers the lattice residue immediately to the right of one selected at random, this second residue also, by definition, is selected at random and thus also has a probability of $\left(1-s C_{\mathrm{b}} / C_{\mathrm{bp}}\right)$ being free. However, due to the natures of the lattice, there are only two ways in which this two-step random selection can be made. One way is that the first residue chosen is free (an $f$ residue chosen with probability $1-s Z$ ) and has a free residue to its right (conditional probability, $f f$ ); the other way is that the first residue chosen is the right end of a bound ligand (a $b_{\mathrm{s}}$ residue chosen with probability $\left.\left(s C_{\mathrm{b}} / C_{\mathrm{bp}}\right) / s=C_{\mathrm{b}} / C_{\mathrm{bp}}\right)$ and has a free residue to its right (conditional probability $b_{s}$ f). Since the over-all probability that the second residue is free must be independent of the method of (random) selection, we obtain:

$$
\left(1-s C_{\mathrm{b}} / C_{\mathrm{bp}}\right)(f f)+C_{\mathrm{b}} / C_{\mathrm{bp}}\left(b_{\mathrm{s}} f\right)=1-s C_{\mathrm{b}} / C_{\mathrm{bp}}
$$

By definition, $\omega$ is the probability of the isolated ligand formerly located has been moved, and is now bound in a single contiguous fashion; or the probability of the single contiguous ligand formerly located has been moved, and is now bound in a double contiguous fashion.

$$
\omega=\frac{\left(b_{\mathrm{s}} b_{1}\right)(f f)}{\left(b_{\mathrm{s}} f\right)\left(f b_{1}\right)}
$$

By rearranging terms among Eqs. (11) - (14), the above expression for $\omega=1$, we get the following expressions for the conditional probabilities:

$$
\begin{aligned}
& (f f)=\left(b_{s} f\right)=\frac{1-s C_{\mathrm{b}} / C_{\mathrm{bp}}}{1-(s-1) C_{\mathrm{b}} / C_{\mathrm{bp}}} \\
& \left(f b_{1}\right)=\left(b_{\mathrm{s}} b_{1}\right)=\frac{C_{\mathrm{b}} / C_{\mathrm{bp}}}{1-(s-1) C_{\mathrm{b}} / C_{\mathrm{bp}}}
\end{aligned}
$$

Introducing Eqs. (15a) and (15b) to (10), and $\omega=1$ being linked, we now have the expression for the mass-action equation:

$$
\frac{C_{\mathrm{b}} / C_{\mathrm{bp}}}{C_{\mathrm{f}}}=K\left(1-s C_{\mathrm{b}} / C_{\mathrm{bp}}\right)\left(\frac{1-s C_{\mathrm{b}} / C_{\mathrm{bp}}}{1-(s-1) C_{\mathrm{b}} / C_{\mathrm{bp}}}\right)^{\mathrm{s}-1}
$$

The volt-ampere characteristics are different before and after the interaction of drug binding with DNA using electrochemistry methods. This study is based on this principle. The ligand both in binding and free state has been assumed to be an electroactive species, and the typical shape of the electrochemical curve has not changed. Thus, one can obtain that the peak current, $i_{\mathrm{p}}$, is given by:

$$
\begin{aligned}
& i_{\mathrm{pb}}=k_{\mathrm{b}} C_{\mathrm{t}} \\
& i_{\mathrm{pf}}=k_{\mathrm{f}} C_{\mathrm{t}}
\end{aligned}
$$

Here $k_{\mathrm{b}}$ and $k_{\mathrm{f}}$ are the heterogeneous rate constants of the DNAdrug adduct and the drug at fixed potential, respectively. The molar fraction of the binding ligand molecules and the total concentration of ligand molecules can be defined as $x$ :

$$
x=\left(i_{\mathrm{pf}}-i_{\mathrm{p}}\right) /\left(i_{\mathrm{pf}}-i_{\mathrm{pb}}\right)
$$

The concentration of ligand can also be expressed as follows by rearranging terms among Eqs. (17) - (19):

$$
\begin{aligned}
& C_{\mathrm{f}}=(1-x) C_{\mathrm{t}}=\frac{\left(i_{\mathrm{p}}-i_{\mathrm{pb}}\right) C_{\mathrm{t}}}{i_{\mathrm{pf}}-i_{\mathrm{pb}}} \\
& C_{\mathrm{b}} / C_{\mathrm{bp}}=x C_{\mathrm{t}} / C_{\mathrm{NP}}=\frac{\left(i_{\mathrm{pf}}-i_{\mathrm{p}}\right) C_{\mathrm{t}}}{\left(i_{\mathrm{pf}}-i_{\mathrm{pb}}\right) C_{\mathrm{bp}}} \\
& \frac{C_{\mathrm{b}} / C_{\mathrm{bp}}}{C_{\mathrm{f}}}=\frac{\left(i_{\mathrm{pf}}-i_{\mathrm{p}}\right)}{\left(i_{\mathrm{p}}-i_{\mathrm{pb}}\right) C_{\mathrm{bp}}}
\end{aligned}
$$

If Eqs. (21) and (22) are introduced into mass-action, the following results can be generalized:

$$
\frac{\left(i_{\mathrm{pf}}-i_{\mathrm{p}}\right)}{\left(i_{\mathrm{p}}-i_{\mathrm{pb}}\right) C_{\mathrm{bP}}}=K\left(1-s \frac{\left(i_{\mathrm{pf}}-i_{\mathrm{p}}\right) C_{\mathrm{t}}}{\left(i_{\mathrm{pf}}-i_{\mathrm{pb}}\right) C_{\mathrm{bP}}}\right)\left(\frac{1-s \frac{\left(i_{\mathrm{pf}}-i_{\mathrm{p}}\right) C_{\mathrm{t}}}{\left(i_{\mathrm{pf}}-i_{\mathrm{pb}}\right) C_{\mathrm{bP}}}}{1-(s-1) \frac{\left(i_{\mathrm{pf}}-i_{\mathrm{p}}\right) C_{\mathrm{t}}}{\left(i_{\mathrm{pf}}-i_{\mathrm{pb}}\right) C_{\mathrm{bP}}}}\right)^{s-1}
$$

The binding constant $K$ and the binding sizes $s$ can be estimated at the same time by using Levenberg-Marquardt algorithm for data fitting by Eq. (23). The $i_{\mathrm{pf}}, i_{\mathrm{pb}}, i_{\mathrm{p}}$ values can be determined by electrochemistry methods, and the plot of ( $i_{\mathrm{pf}}$ $\left.-i_{\mathrm{p}}\right) C_{\mathrm{t}} /\left(i_{\mathrm{pf}}-i_{\mathrm{pb}}\right) C_{\mathrm{bp}} v s .\left(i_{\mathrm{pf}}-i_{\mathrm{p}}\right) /\left(i_{\mathrm{p}}-i_{\mathrm{pb}}\right) C_{\mathrm{bp}}$ can be displayed.

\section{Experimental}

\section{Apparatus and reagents}

The DPV experiment were carried out in a CHI660A electrochemical analyzer ( $\mathrm{CH}$ Instruments, USA). The working electrode was an oxidized waxed graphite disk electrode with a geometric area of $0.324 \mathrm{~cm}^{2}$. $\mathrm{An} \mathrm{Ag} / \mathrm{AgCl} / \mathrm{KCl}$ electrode was used as the reference electrode, and a platinum wire was used as the counter electrode.

ctDNA (Sigma Chemical Company) and Gatifloxacin (Huamei Biotechnological, Shanghai) were purchased. The supporting electrolyte of DPV experiments was $0.05 \mathrm{~mol} \mathrm{~L}^{-1}$ Tris- $\mathrm{HCl}$ buffer of $\mathrm{pH} 7.2\left(0.1 \mathrm{~mol} \mathrm{~L}^{-1} \mathrm{NaCl}\right.$ was used to keep the ionic strength constant). All other regents were of analytical grade and doubly distilled water was used throughout.

\section{Procedures}

All voltammetric experiments were carried out in a singlecompartment cell of volume $10 \mathrm{ml}$. The working electrode was polished with 0.05 alumina suspension to a mirror finish. The electrode was then washed with distilled water, and was oxidized at $1.7 \mathrm{~V}$ for $60 \mathrm{~s}$ before each measurement.

(1) $2.0 \times 10^{-4} \mathrm{~mol} \mathrm{~L}^{-1}$ GTFX samples were diluted with Tris- $\mathrm{HCl}$ buffer; the serial concentrations of solutions for free GTFX $\left(1.0 \times 10^{-5} \mathrm{~mol} \mathrm{~L}^{-1}\right): 0.40,0.60,0.80,1.20,1.60,2.00$, $2.40,2.80,3.20,3.40,3.60,3.80$ and 4.00 were obtained.

(2) $1.847 \times 10^{-3} \mathrm{~mol} \mathrm{~L}^{-1} \mathrm{ctDNA}$ was considered as mother 
Table 1 Relationships between peak current and concentration of GTFX and ctDNA

\begin{tabular}{cccccccc}
\hline$C_{\mathrm{t}} / 10^{-5} \mathrm{~mol} \mathrm{~L}^{-1}$ & $i_{\mathrm{pf}} / \mu \mathrm{A}$ & $\begin{array}{c}\text { Adding } \\
C_{\mathrm{DNA}} \text { mol L-1 } \\
\text { (non-saturation) }\end{array}$ & $i_{\mathrm{p}} / \mu \mathrm{A}$ & $\begin{array}{c}\text { Adding } \\
C_{\mathrm{DNA}} \text { mol L-1 } \\
(\text { saturation) }\end{array}$ & $i_{\mathrm{pb}} / \mu \mathrm{A}$ & $\frac{\left(i_{\mathrm{pf}}-i_{\mathrm{p}}\right) C_{\mathrm{t}}}{\left(i_{\mathrm{pf}}-i_{\mathrm{pb}}\right) C_{\mathrm{bp}}}$ & $\frac{\left(i_{\mathrm{pf}}-i_{\mathrm{p}}\right)}{\left(i_{\mathrm{p}}-i_{\mathrm{pb}}\right) C_{\mathrm{bp}}}$ \\
\hline 0.40 & 0.782 & $5.167 \times 10^{-5}$ & 0.342 & $1.773 \times 10^{-3}$ & 0.266 & 0.0661 & $1.1205 \times 10^{5}$ \\
0.60 & 1.047 & $5.167 \times 10^{-5}$ & 0.522 & $1.754 \times 10^{-3}$ & 0.413 & 0.0962 & $9.3217 \times 10^{4}$ \\
0.80 & 1.278 & $5.167 \times 10^{-5}$ & 0.708 & $1.736 \times 10^{-3}$ & 0.561 & 0.1231 & $7.5045 \times 10^{4}$ \\
1.20 & 1.981 & $5.167 \times 10^{-5}$ & 1.101 & $1.699 \times 10^{-3}$ & 0.741 & 0.1648 & $4.7309 \times 10^{4}$ \\
1.60 & 2.547 & $5.167 \times 10^{-5}$ & 1.582 & $1.662 \times 10^{-3}$ & 0.986 & 0.1914 & $3.1336 \times 10^{4}$ \\
2.00 & 3.102 & $5.167 \times 10^{-5}$ & 2.094 & $1.625 \times 10^{-3}$ & 1.210 & 0.2062 & $2.2069 \times 10^{4}$ \\
2.40 & 3.683 & $5.167 \times 10^{-5}$ & 2.648 & $1.588 \times 10^{-3}$ & 1.442 & 0.2145 & $1.6609 \times 10^{4}$ \\
2.80 & 4.386 & $5.167 \times 10^{-5}$ & 3.272 & $1.551 \times 10^{-3}$ & 1.681 & 0.2232 & $1.3552 \times 10^{4}$ \\
3.20 & 4.973 & $5.167 \times 10^{-5}$ & 3.822 & $1.514 \times 10^{-3}$ & 1.999 & 0.2397 & $1.2219 \times 10^{4}$ \\
3.40 & 5.296 & $5.167 \times 10^{-5}$ & 4.143 & $1.496 \times 10^{-3}$ & 2.132 & 0.2398 & $1.1097 \times 10^{4}$ \\
3.60 & 5.584 & $5.167 \times 10^{-5}$ & 4.425 & $1.478 \times 10^{-3}$ & 2.274 & 0.2440 & $1.0428 \times 10^{4}$ \\
3.80 & 5.921 & $5.167 \times 10^{-5}$ & 4.768 & $1.459 \times 10^{-3}$ & 2.487 & 0.2469 & $9.7829 \times 10^{3}$ \\
4.00 & 6.528 & $5.167 \times 10^{-5}$ & 5.215 & $1.454 \times 10^{-3}$ & 2.552 & 0.2557 & $9.5424 \times 10^{3}$ \\
\hline
\end{tabular}

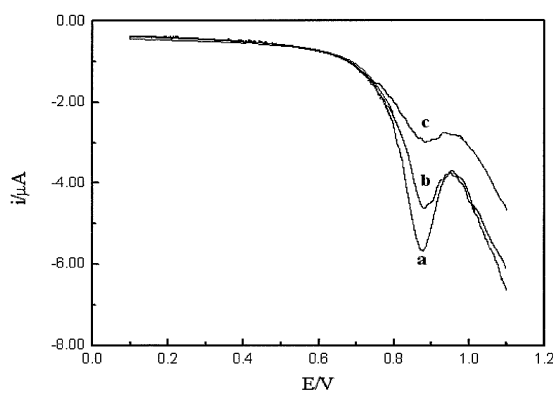

Fig. 1 Different pulse voltammograms of GTFX interaction with ctDNA. Scan rate, $20 \mathrm{mV} / \mathrm{s}$; pulse width, $50 \mathrm{~ms}$. a, $2.0 \times 10^{-5} \mathrm{~mol}$ $\mathrm{L}^{-1}$ GTFX; b, $2.0 \times 10^{-5} \mathrm{~mol} \mathrm{~L}^{-1} \mathrm{GTFX}+4.5 \times 10^{-5} \mathrm{~mol} \mathrm{~L}^{-1} \mathrm{ctDNA}$; c, $2.0 \times 10^{-5} \mathrm{~mol} \mathrm{~L}^{-1} \mathrm{GTFX}+1.0 \times 10^{-3} \mathrm{~mol} \mathrm{~L}^{-1} \mathrm{ctDNA}$.

liquor, and was added to each numbered cell, which contained GTFX solutions (the concentration is from low to high, corresponding (1)). The concentrations of ctDNA $\left(1.0 \times 10^{-5}\right.$ mol L $\left.{ }^{-1}\right): 1.773,1.754,1.736,1.699,1.662$, 1.625, 1.588, 1.551, $1.514,1.496,1.478,1.459,1.454$ were reached and the serial concentrations of solutions for binding drug were prepared.

(3) The preparation of drug and drug-DNA adduct coexist in solution: $1.292 \times 10^{-3} \mathrm{~mol} \mathrm{~L}^{-1} \mathrm{ctDNA}$ was added to each numbered cell, which contained GTFX solutions (the concentration is from low to high, corresponding (1)). The final concentration of ctDNA was $5.167 \times 10^{-5} \mathrm{~mol} \mathrm{~L}^{-1}$ in each cell.

These prepared solutions were stirred adequately for a given period, say $30 \mathrm{~min}$ or more. Electrochemical characterization of drug and the interaction of drug-DNA were investigated by DPV. Where appropriate, solutions were deoxygenated via purging with $\mathrm{N}_{2}$ gas for 10 min prior to measurements.

All measurements were carried out at ambient temperature of the laboratory $\left(23-27^{\circ} \mathrm{C}\right)$. All data, unless specified otherwise, were the averages of three to five repeated measurements.

The ORIGIN software was used for regression analysis and data fitting based on experimental determination.

\section{Results and Discussion}

\section{Electrochemical behavior of GTFX}

No response occurred at DPV using a general solid electrode, such as platinum, gold, or glassy-carbon (The same result was obtained for the $\mathrm{CV}$ method including a carbon paraffined electrode). While the oxidized graphite electrode was used, the typical DPV behavior of GTFX in the absence and presence of ctDNA appeared as shown in Fig. 1. In the absence of DNA, it could be seen that a sensitive oxidation peak appears at $0.88 \mathrm{~V}$ (curve a in Fig. 1). In the presence of DNA, the corresponding peak current decreased apparently (curve b in Fig. 1), and addition of excess of DNA in the GTFX solution causes the peak currents to diminish considerably (curve $\mathrm{c}$ in Fig. 1), but the peak potential has no significant shift. The researches have previously indicated that the decreasing of peaks is due to the interaction of drug and DNA, and not to the increased viscosity of the solution or the blockage of the electrode surface by DNA adsorption, ${ }^{16,17}$ a special experiment designed in the literature indicated that the addition of DNA affects the current only slightly. Thus, according to these observations, it seems that the decreases of peak currents of GTFX after an addition of DNA are caused by intercalation of GTFX to the bulky, slowly diffusing DNA, which results in a considerable decrease in the apparent diffusion coefficient.

\section{Interaction of GTFX and DNA}

The DPV determination shows that the interaction of GTFX and DNA occurred in buffer solution. The peak currents obtained for GTFX and GTFX-DNA adduct show linear dependence on the GTFX concentration in the cell. Plots of $i_{\mathrm{p}}$ vs. $c$ are shown in Fig. 2 for GTFX and GTFX-DNA adduct, the GTFX concentration for this calibration curve ranging from 4.0 $\times 10^{-6} \mathrm{~mol} \mathrm{~L}^{-1}$ to $4.0 \times 10^{-5} \mathrm{~mol} \mathrm{~L}^{-1}$.

In the above concentration scope, the peak currents of GTFX and GTFX-DNA adduct are measured and the experimental data are shown in Table 1. A serial number of $\left(i_{\mathrm{pf}}-i_{\mathrm{p}}\right) C_{\mathrm{t}} /\left(i_{\mathrm{pf}}-i_{\mathrm{pb}}\right) C_{\mathrm{bp}}$ $(x),\left(i_{\mathrm{pf}}-i_{\mathrm{p}}\right) /\left(i_{\mathrm{p}}-i_{\mathrm{pb}}\right) C_{\mathrm{bp}}(y)$ could be gained from Table 1 . A nonlinear fit analysis of the data $(x-y)$ to Eq. (23) yielded the binding curves shown in Fig. 3 and the following parameters were obtained: $K=1.7058 \times 10^{5}\left(\mathrm{~mol} \mathrm{~L}^{-1}\right)^{-1}, \quad s=3.09$ (correlation coefficient $R^{2}=0.9959$ ).

\section{Conclusions}

In this paper, we report the DPV behavior of GTFX and its binding with natural ctDNA at a carbon paraffined electrode. A practical electrochemical equation, which is rebuilt from the McGhee model, is used to examine the interaction of this kind 


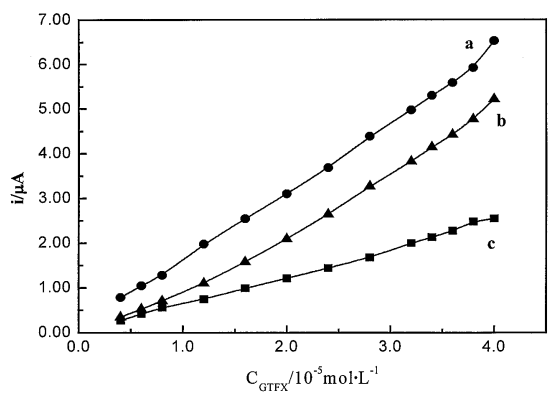

Fig. 2 Calibration curve of $\left(i_{\mathrm{p}}\right)-(c)$. a, GTFX; b, GTFX +1.2918 $\times 10^{-3} \mathrm{~mol} \mathrm{~L}^{-1} \mathrm{ctDNA} ; \mathrm{c}$, GTFX $+1.8464 \times 10^{-3} \mathrm{~mol} \mathrm{~L}-1 \mathrm{ctDNA}$.

of drug. Based on the equation, the binding constant $(K)$ and the size of binding sites $s$ (base pairs) are simultaneously obtained from the dependence of the current on the amount of added DNA in different pulse voltammetry measurements. The interaction profile could be drawn quickly in this way. This research provides a convenient and sensitive method, and offers great promise for further study of interaction between DNA and electroactive ligands.

In this research, the binding constant of the GTFX-DNA complex formation is estimated as $1.7058 \times 10^{5}\left(\mathrm{~mol} \mathrm{~L}^{-1}\right)^{-1}$, and 3 base pairs of DNA are covered by each GTFX molecule, which suggests a moderate strength binding reaction between GTFX and ctDNA. This result, with the help of other investigator's experiments, ${ }^{17-21}$ demonstrates that GTFX has the properties of an intercalative binder, and the electrostatic interaction should be the driving source except for the nonspecific binding.

\section{References}

1. J. M. Blondeau, Clinical Therapeutics, 1999, 21, 3.

2. M. Nakashima, T. Uematsu, K. Kosuge, H. Kusajima, T. Dori, Y. Masuda, R. Ishida, and H. Uchida, Antimicrob. Agents Chemother., 1995, 39, 2635.

3. Y. Muraki, M. Yamada, S. Nii, H. Kumon, and H. Ohmori, Jpn. J. Chemother., 1995, 43, 357.

4. A. G. Krishna, D. V. Kumar, B. M. Khan, S. K. Rawal, and K. N. Ganesh, Biochim. Biophys. Acta, 1998, 1381, 104.

5. X. G. Qu, C. Z. Wan, H. C. Becker, D. P. Zhong, and A. H. Zewail, Proc. Natl. Acad. Sci. USA, 2001, 98, 14212.

6. A. M. Pyle, J. P. Rehmann, R. Meshoyrer, C. V. Kumar, N. J. Turro, and J. K. Barton, J. Am. Chem. Soc., 1989, 111,

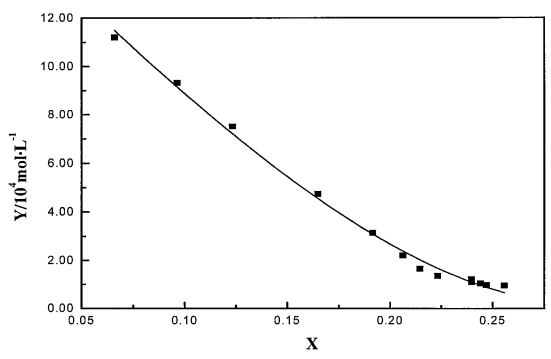

Fig. 3 Binding curve of GTFX with ctDNA in $0.05 \mathrm{~mol} \mathrm{~L}^{-1} \mathrm{HCl}$ Tris solution. $x=\left(i_{\mathrm{pf}}-i_{\mathrm{p}}\right) C_{\mathrm{t}} /\left(i_{\mathrm{pf}}-i_{\mathrm{pb}}\right) C_{\mathrm{bp}}, y=\left(i_{\mathrm{pf}}-i_{\mathrm{p}}\right) /\left(i_{\mathrm{p}}-i_{\mathrm{pb}}\right) C_{\mathrm{bp}}$.

3051.

7. C. V. Kumar and E. H. Asuncion, J. Am. Chem. Soc., 1993, $115,8547$.

8. G. S. Kumar, S. M. Musser, J. Cummings, and Z. M. Tomas, J. Am. Chem. Soc., 1996, 118, 9209.

9. F. F. Leng, W. Priebe, and J. B. Chaires, Biochemistry, 1998, 37, 1743.

10. Y. Maeda, K. Nunomura, and E. Ohtsubo, J. Mol. Biol., 1990, 215, 321.

11. J. D. McGhee and P. H. Von-Hippel, J. Mol. Biol., 1974, 86,469

12. K. M. Millan and S. R. Mikkelsen, Anal. Chem., 1993, 65, 2317.

13. P. Yang, H. F. Wang, and B. S. Yang, Chem. J. Chin Univ., 1995, 16, 1075

14. S. F. Wang, Doctoral Dissertation, Zhejiang University, 2003.

15. J. K. Barton, J. M. Goldberg, C. V. Kumar, and N. J. Turro, J. Am. Chem. Soc., 1986, 108, 2081.

16. X. Chu, G. F. Shen, J. H. Jiang, T. F. Kang, B. Xiong, and R. Q. Yu, Anal. Chim. Acta, 1998, 373, 29.

17. S. F. Wang, T. Z. Peng, and C. F. Yang, J. Biochem. Biophys. Methods, 2003, 55, 191.

18. I. D. Vilfan, P. Drevenšek, I. Turel, and N. P. Ulrih, Biochim. Biophys. Acta, 2003, 1628, 111.

19. G. S. Son, J. A. Yeo, M. S. Kim, S. K. Kim, A. Holmén, B. Åkerman, and B. Nordén, J. Am. Chem. Soc., 1998, 120, 6451.

20. H. J. Hwangbo, Y. A. Lee, J. H. Park, Y. R. Lee, J. M. Kim, S. Y. Yi, and S. K. Kim, Bull. Korean Chem. Soc., 2003, 24, 579.

21. G. Palú, G. Valisena, G. Ciarrocchi, B. Gatto, and M. Palumbo, Proc. Natl. Acad. Sci. U. S. A., 1992, 89, 9671. 